\title{
How do you feel? A warm and touching 2021 Nobel tribute
}

\author{
Michael J. Caterina
}

Departments of Neurosurgery, Biological Chemistry, and Neuroscience, Neurosurgery Pain Research Institute, Johns Hopkins University School of Medicine, Baltimore, Maryland, USA.

$\mathbf{P}$ erceptions of touch, temperature, and pain inform us about our bodies' condition and the world around us, and are critical determinants of our function, self-protection, comfort, and social interactions. Charles Sherrington, 1932 Nobel laureate, proposed that our perception of physically diverse stimuli over different intensities as distinct sensations implied the existence of correspondingly distinct neuronal mechanisms to detect these stimuli (1). Studies in the mid-1900s confirmed the existence of distinct peripheral somatosensory neuron subpopulations differentially tuned to touch, temperature, and irritant chemicals that produce a range of innocuous and noxious perceptions. However, the molecular mechanisms by which such physically distinct stimuli are detected and quantified remained enigmatic for several more decades. The 2021 Nobel Prize in Physiology or Medicine, which was shared by David Julius at the University of California, San Francisco, and Ardem Patapoutian at the Scripps Research Institute, fittingly recognized their breakthrough discoveries that helped to fill this critical gap in knowledge.

\section{Thermomimetic chemicals aid the discovery of thermosensitive ion channels}

Spicy foods containing the pungent chemical capsaicin are dietary staples worldwide. The burning sensation produced by capsaicin is its most familiar and celebrated trait, and one that is also exploited for such purposes as self-defense and bird feeder squirrel deterrence. However, capsaicin can also paradoxically desensitize the nervous system to capsaicin itself and to other painful stimuli like heat. This phenomenon has led to the medicinal use of capsaicin as an analgesic. It also allowed Jancso, Szolcsanyi, and colleagues in the 1950 s and 1960s to recognize that capsaicin causes selective degeneration of a specific subset of presumably pain-initiating (i.e., nociceptive) sensory neurons. Szallasi and Blumberg subsequently showed that nociceptive neurons express a discrete binding site for capsaicin and related compounds. Because these compounds share a homovanillic acid chemical moiety, they dubbed this site a vanilloid receptor. Other investigators reported that capsaicin could activate an ion channel current in cultured nociceptive sensory neurons. Yet, the molecular nature of the vanilloid receptor and its relationship to the channels involved was unclear (2).

It was in this setting that the lab of David Julius, a pioneer in the use of expression cloning to identify elusive neurotransmitter receptors, sought to clone the vanilloid receptor. As a postdoctoral fellow in the Julius lab at the time, I was privileged to be a part of the team that accomplished this goal. Based on the assumption that the vanilloid receptor would mediate calcium influx when activated, our team introduced complex pools of sensory ganglion-derived cDNAs into HEK293 cells, kidney-derived cells that lack capsaicin responsiveness. Using a highly optimized fluorescent calcium imaging assay, we identified a single cDNA clone that was sufficient to confer capsaicin-induced calcium influx into these cells (3). Examination of the encoded protein sequence revealed it to be related to transient receptor potential (TRP) ion channels, key participants in phototransduction in the Drosophila eye. On this basis, the newly cloned vanilloid receptor was later renamed TRP vanilloid 1 (TRPV1). Another notable fea-
Conflict of interest: MJC was an inventor on a former patent (US patent $6,335,180$ ) on TRPV1 and TRPV2 that was licensed through UCSF and Merck, and was entitled to royalties on that patent.

Copyright: (c) 2021, American Society for Clinical Investigation.

Reference information: J Clin Invest. 2021;131(24):e156587. https://doi.org/10.1172/JCI156587. ture of TRPV1 was that its expression was highly enriched in dorsal root and trigeminal sensory ganglia, and in particular in small-to-medium diameter nociceptive neurons, offering a compelling potential basis for capsaicin-evoked pain (3).

While this finding addressed a long-standing question in sensory biology, capsaicin is not a normal constituent of our bodies. Therefore, it remained unclear what the physiological function of TRPV1 might be. To address this question, the Julius team began to screen for alternative TRPV1 activators. One commonly encountered painful chemical stimulus that proved capable of activating TRPV1 was acid (4). However, an even more striking finding was that TRPV1 could be activated simply by increasing ambient temperature to levels that normally cause pain (i.e., $>45^{\circ} \mathrm{C}$ ) (3). Other investigators had previously demonstrated that heat could activate cationic channel currents in cultured sensory neurons, but not sympathetic neurons (5, 6). The finding that TRPV1 could be gated by temperature suggested that it might be one mediator of this "molecular thermometer" function. A satisfying confirmation of this notion came several years later, when we analyzed mice in which the TRPV1 gene was disrupted. Those mice showed not only a complete absence of responsiveness to capsaicin but also a partial reduction in heat-evoked pain behaviors and a substantially reduced level of heat hypersensitivity in the setting of skin inflammation (7). These findings thus confirmed a fundamental role for TRPV1 in heat pain, and in doing so explained the similarity between the piquante "burn" of chili peppers and the caliente burn of a hot flame.

The labs of Julius, Patapoutian, and others subsequently identified additional TRP channels that could be gated by mild or intense heat, and/or by irritant chemicals. However, the full generalization of TRP channels as thermosensors (and the broader value of thermomimetic chemi- 

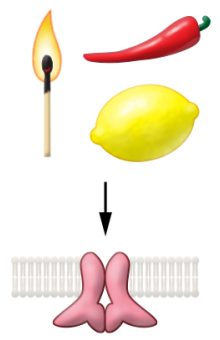

TRPV1

Pungency

Burning pain Warmth?
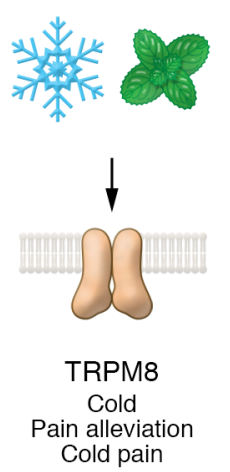

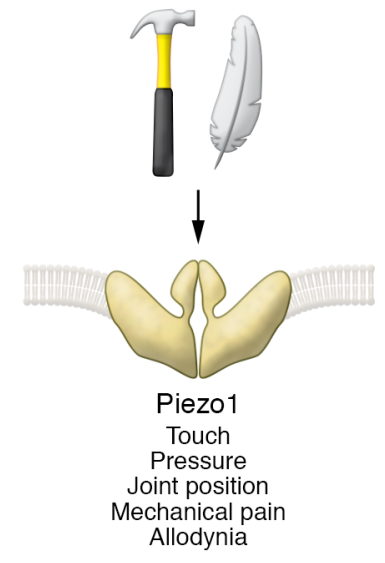

Figure 1. Sensory receptor molecules exhibit diverse inputs and outputs. Top: Physical and chemical stimuli detected by the ion channel receptors identified by Julius and Patapoutian (match, painful heat; pepper, capsaicin; lemon, acid; snowflake, cold; mint leaves, menthol; hammer, strong mechanical force; feather, weak mechanical force). Middle: Cartoons of TRPV1, TRPM8, and Piezo ion channel structures. Bottom: Sensory perceptions evoked through each channel.

cals) became apparent in 2002, when the Julius and Patapoutian labs in parallel published on the cloning and characterization of yet another temperature-gated TRP channel, TRPM8. This channel, intriguingly, was gated by cold, rather than hot temperatures, and could alternatively be activated by menthol and related compounds that produce a cooling sensation $(8,9)$. As with TRPV1, evidence for the physiological importance of TRPM8 came from the study of knockout mice, which exhibited virtual elimination of behavioral and neurophysiological responses to mildly cold temperatures (10-12). The relationship between TRPM8 and pain appears to be both complex and context dependent.

\section{Loss-of-function strategy uncovers identity of receptors for touch}

Physiological studies prior to 2010 revealed the existence and functional properties of mechanically activated channels in both vertebrate and invertebrate species. Among the best studied of these were the mechanoreceptors of the vertebrate inner ear and bacterial Msc mechanically gated ion channels. A number of channels with mechanosensory or osmosensory properties (including members of the two-pore potassium, degenerin/ ENaC, and TRP channel families, among others) were also identified in sensory neurons (13). However, despite all that had been learned about these channels and the heterogeneous mechanosensory characteristics of peripheral somatosensory neurons, the molecular identity of the primary molecule(s) mediating acute mechanosensation in low-threshold mechanoreceptors and nociceptors in mammals remained elusive. In contrast to the situation with thermosensation, there also were no chemical agents available that directly mimicked the perception of touch. In the absence of such tools, the Patapoutian lab adopted a painstaking but powerful lossof-function strategy to discover candidate mechanoreceptor molecules. They first identified N2A mouse neuroblastoma cells as an easily transfected cell line exhibiting a strong mechanosensory response in voltage-clamp experiments. With these cells in hand, Patapoutian and colleagues systematically knocked down the expression of individual mRNAs expressed in N2A cells that were predicted to encode membrane proteins with channel-like characteristics. This screen led to the identification of a single mRNA whose knockdown suppressed the mechanically evoked currents in N2A cells. Furthermore, the heterologous expression of this mRNA introduced a mechanically gated ion channel current in multiple cell types. Based on these demonstrations of necessity and sufficiency, Patapoutian and colleagues designated the encoded protein Piezo1, which is derived from the Greek word for pressure, piesi. One remarkable feature carried out an siRNA screen, in which they of Piezo1 was its extraordinary size. The protein was predicted to contain 24 to 36 transmembrane domains, making it one of the largest and most complex membrane polypeptides known (14).

While Piezo1 appeared not to be prominently expressed in sensory ganglia, a homologous protein, Piezo2, was expressed at high levels in that tissue, making it a promising candidate for somatosensory mechanotransduction. Indeed, siRNA-mediated depletion of Piezo2 from cultured sensory neurons reduced the prevalence of mechanosensory currents in these cells (14). Moreover, subsequent knockout experiments conducted by Patapoutian and colleagues in mice and fruit flies provided overwhelming in vivo support for the importance of Piezo2 and the Drosophila Piezo ortholog to mechanosensory neuron function. For example, mice in which Piezo2 was eliminated from caudal sensory neurons exhibited virtually no behavioral responses to light touch, partially impaired responses to painful mechanical stimuli, and a dramatic reduction in mechanical allodynia following capsaicin injection or nerve injury (15). The importance of Piezo proteins to mechanotransduction is not confined to model organisms. Human subjects with rare Piezo2 loss-of-function mutations were found to have deficits in both light touch sensation and proprioception, while those with gain-of-function Piezo2 mutations exhibit arthrogryposis, a disorder featuring immobilizing contracture of joints. Examination of Piezo1- and Piezo2-knockout mice has also revealed functions in mechanosensory processes in numerous other tissues. In the cardiovascular system, for example, these include roles in endothelial cell organization, vascular tone, vascular remodeling, red blood cell volume regulation, and blood pressure regulation. In the lung, absence of Piezo2 from sensory neurons resulted in an impaired stretch-evoked breathing reflex (16). Thus, the importance of Piezo-mediated mechanotransduction extends far beyond touch and pain.

\section{Molecular structures and biomedical implications}

In addition to their discoveries of thermosensory and mechanosensory channels and the demonstration of the importance 
of these channels to sensory function, Julius, Patapoutian, and their colleagues also made landmark discoveries on the biophysical front, through their use of cryo-electron microscopy (cryo-EM) to reveal the structures of these remarkable molecular machines and the multiple mechanisms by which they are activated (17-19). These studies were greatly accelerated by a collaboration between Julius and University of California, San Francisco, colleague Yifan Cheng, in which they applied powerful refinements to the cryoEM method, allowing visualization of the complex TRPV1 macromolecular structure at unprecedented resolution and in discrete functional states $(17,18)$. From a technical perspective, these studies helped to usher in a revolution in membrane protein biophysics.

The potential medical breakthroughs enabled by the discoveries of temperature- and touch-gated channels are enormous, but remain to be fully realized. For example, while many pharmaceutical companies have developed potent and selective antagonists against TRPV1, the tendencies of these antagonists to block desirable heat avoidance reflexes and of some of them to produce a transient fever have impeded their clinical advancement (20). The complexity of the Piezo channels has also apparently presented challenges to the development of potent and selective antagonists. However, as understanding of both classes of molecules advances, with the help of the aforementioned cryo-EM studies, it seems possible that rationally designed modulators might emerge that overcome these challenges and could be used to treat conditions such as chronic pain or cardiovascular disease. In addition, detailed understanding of the modulation of these proteins during pathological conditions could lead to the development of therapies that spare their desirable physiological functions but sup- press their augmented activities during pathological pain states.

At a more fundamental scientific level, in addition to answering longstanding questions about our perception of the world around us, the discovery and characterization of thermosensory TRP channels and mechanosensory Piezo channels have revealed them to be, in some ways, molecular microcosms of the sensory nervous system. Amazingly, these individual proteins are capable of carrying out physical signal detection and measurement, signal discrimination, and (in the case of TRPV1 and TRPM8) polymodal sensitivity (Figure 1), as well as exhibiting desensitization and sensitization phenomena. All of these are complex traits of the peripheral sensory neurons in which the channels reside and of the sensory nervous system as a whole. It is worth noting, however, that these transduction molecules cannot alone account for the emergent properties of our complex sensory nervous system.

Finally, the broad sweep of discoveries carried out by the Julius and Patapoutian labs on these channels, ranging from atomic-level mechanism to in vivo physiological and pathophysiological function, illustrates the power of innovative, interdisciplinary basic scientific inquiry to solve outstanding biomedical problems.

\section{Acknowledgments}

MJC is supported by grants NS103974, AR072230, and DE027731 from the NIH, and the Neurosurgery Pain Research Institute at Johns Hopkins.

Address correspondence to: Michael J. Caterina, 725 North Wolfe St., Baltimore, Maryland 21205, USA. Phone: 410.502.5457; Email: caterina@jhmi.edu.

1. Sherrington CS. The Integrative Action of the Nervous System. Yale University Press; 1906.

2. Szallasi A, Blumberg PM. Vanilloid (capsaicin) receptors and mechanisms. Pharmacol Rev.
1999;51(2):159-212.

3. Caterina MJ, et al. The capsaicin receptor: a heat-activated ion channel in the pain pathway. Nature. 1997;389(6653):816-824.

4. Tominaga $\mathrm{M}$, et al. The cloned capsaicin receptor integrates multiple pain-producing stimuli. Neuron. 1998;21(3):1-543.

5. Cesare P, McNaughton P. A novel heat-activated current in nociceptive neurons and its sensitization by bradykinin. Proc Natl Acad Sci U S A. 1996;93(26):15435-15439.

6. Reichling DB, Levine JD. Heat transduction in rat sensory neurons by calcium-dependent activation of a cation channel. Proc Natl Acad Sci US A. 1997;94(13):7006-7011.

7. Caterina MJ, et al. Impaired nociception and pain sensation in mice lacking the capsaicin receptor. Science. 2000;288(5464):306-313.

8. McKemy DD, et al. Identification of a cold receptor reveals a general role for TRP channels in thermosensation. Nature. 2002;416(6876):52-58.

9. Peier AM, et al. A TRP channel that senses cold stimuli and menthol. Cell. 2002;108(5):705-715.

10. Bautista DM, et al. The menthol receptor TRPM8 is the principal detector of environmental cold. Nature. 2007;448(7150):204-208.

11. Colburn RW, et al. Attenuated cold sensitivity in TRPM8 null mice. Neuron. 2007;54(3):379-386.

12. Dhaka A, et al. TRPM8 is required for cold sensation in mice. Neuron. 2007;54(3):371-378.

13. Marshall KL, Lumpkin EA. The molecular basis of mechanosensory transduction. Adv Exp Med Biol. 2012;739:142-155.

14. Coste B, et al. Piezo1 and Piezo2 are essential components of distinct mechanically activated cation channels. Science. 2010;330(6000):55-60.

15. Murthy SE, et al. The mechanosensitive ion channel Piezo2 mediates sensitivity to mechanical pain in mice. Sci Transl Med. 2018;10(462):eaat9897.

16. Murthy SE, et al. Piezos thrive under pressure: mechanically activated ion channels in health and disease. Nat Rev Mol Cell Biol. 2017;18(12):771-783.

17. Cao E, et al. TRPV1 structures in distinct conformations reveal activation mechanisms. Nature. 2013;504(7478):113-118.

18. Liao M, et al. Structure of the TRPV1 ion channel determined by electron cryo-microscopy. Nature. 2013;504(7478):107-112.

19. Saotome K, et al. Structure of the mechanically activated ion channel Piezo1. Nature. 2018;554(7693):481-486.

20. Moran MM. TRP channels as potential drug targets. Annu Rev Pharmacol Toxicol. 2018;58:309-330. 